Check for updates

Cite this: RSC Adv., 2019, 9, 17691

Received 8th February 2019

Accepted 18th May 2019

DOI: 10.1039/c9ra01027k

rsc.li/rsc-advances

\section{Preparation and application of solid-state upconversion materials based on sodium polyacrylate $\uparrow$}

\author{
Changqing Ye, Jinsuo Ma, (D) Pengju Han, (D) * Shuoran Chen, (D) Ping Ding, Bin Sun \\ and Xiaomei Wang*
}

By loading a microemulsion containing both sensitizer and emitter into porous sodium polyacrylate (PAAS), a water-absorbent resin (WAR) upconversion (UC) material was fabricated for photocatalysis applications. This WAR UC material showed a highly efficient UC process in the ambient environment owing to its liquid/solid encapsulation structure. In the application measurement, the UC emission from WAR UC materials can excite the catalyst $\mathrm{Pt} / \mathrm{WO}_{3}$ to produce hydroxyl radicals, yielding 7-hydroxycoumarin by reacting with coumarin. In another case, since the band gap of $\mathrm{ZnCdS}$ matches the energy of UC emission, hole-electron pairs can be obtained under the UC irradiation and capture electrons from rhodamine $\mathrm{B}$, leading to the degradation of rhodamine $\mathrm{B}$. The maximum of the photocatalysis efficiency can be up to $97 \%$. This work solves the oxygen quenching problem by preparing a triplet-triplet annihilation upconversion (TTA-UC) O/W microemulsion and loading it into PAAS WAR, and opens a new avenue to solid-state devices for TTA-UC. The applications of photocatalytic synthesis and photocatalytic degradation lay a foundation for future practical applications for TTA-UC materials.

\section{Introduction}

Upconversion is a phenomenon of lower-energy (longer wavelength) photons converted into higher-energy photons (shorter wavelength), which can be carried out by either two-photon absorption excited by the illuminant with high power density $^{1-3}$ or triplet-triplet annihilation (TTA) excited with low power density. ${ }^{4-7}$ Owing to the potential widespread practical applications such as in photovoltaics, ${ }^{\mathbf{8} 9}$ photocatalysis ${ }^{10,11} /$ photodegradation, ${ }^{12,13}$ photodynamic therapy of cancer ${ }^{14,15}$ and displays, ${ }^{\mathbf{1 6}}$ triplet-triplet annihilation upconversion (TTA-UC) has attracted more attention.

A TTA-UC system mainly consists of a triplet sensitizer and annihilator, ${ }^{17-19}$ and the UC mechanism can be divided into several micro-channel processes as follows: ${ }^{20-22}$ Upon excitation of the low-energy illuminant, the triplet sensitizer will achieve its singlet state $\left({ }^{1} \mathrm{~S}^{*}\right)$, followed by an intersystem crossing process to reach the triplet state $\left({ }^{3} S^{*}\right)$. Secondly, the energy will be transferred to the annihilator to generate its triplet state $\left({ }^{3} \mathrm{~A}^{*}\right)$ via triplet-triplet energy transfer. Thirdly, two triplet state annihilator will further annihilate by collision and populate the

Research Centre for Green Printing Nanophotonic Materials, Jiangsu Key Laboratory for Environmental Functional Materials, Institute of Chemistry, Biology and Materials Engineering, Suzhou University of Science and Technology, Suzhou 215009, P. R. China. E-mail: wxyhpj@163.com

$\dagger$ Electronic supplementary information (ESI) available. See DOI: 10.1039/c9ra01027k singlet-excited state. This annihilator at the singlet-excited state could ultimately go back to the ground state with UC fluorescence. ${ }^{23}$

Researches of TTA-UC material were generally carried on within solution system ever since proposed in $1962 .{ }^{24}$ Moreover, the mechanism of TTA-UC is based on the triplet population, triplet energy transfer and triplet annihilation process of organic compounds, so the system should be isolated from oxygen to avoid the quenching of triplet excitons. ${ }^{25-27}$ Inevitably, necessity of anaerobic environment and solution system significantly restricts the practical application of TTA-UC. ${ }^{28}$ Thus, high-efficiency TTA-UC in the ambient environment has attracted great interest and been explored by many researchers. For example, sensitizers and annihilators were embedded into solid polymer films ${ }^{29,30}$ or nano particles, ${ }^{31}$ including ethyleneoxide-epichlorohydrin copolymer ${ }^{32}$ with low glass transition temperature or rigid polymethyl methacrylate. ${ }^{33}$ The encapsulation of sensitizers and annihilators could produce anaerobic environment for TTA-UC. However, the triplet-triplet energy transfer and TTA processes of TTA-UC were somewhat suffocated as the diffusion of sensitizers and annihilators restricted by polymer encapsulation and in consequence leading to the significantly decreased quantum efficiency. ${ }^{34,35}$ So, compared with organic solvents system, these strategies all involved complex preparation process and low UC efficiency, ${ }^{36}$ which made TTA-UC difficult for further applications.

For above concerns, we explored a new liquid/solid encapsulation strategy for developing high TTA-UC system. First, we 
utilized an o/w microemulsion which can act as an effective medium for TTA-UC performance in ambient air environment to dissolve the sensitizer PdTPP and the annihilator DPA. Then the $\mathrm{O} / \mathrm{W}$ microemulsion was further encapsulated into a waterabsorbent resin (WAR) of the porous sodium polyacrylate (PAAS) to fabricate solid-state UC material, which can realized highly efficient solid-state TTA-UC in practical ambient environment. This WAR solid-state UC material has been demonstrated to be applied in photocatalytic synthesis of 7hydroxycoumarin and photocatalytic degradation of rhodamine $\mathrm{B}$, which could lay a foundation for future practical effective energy utilization applications for TTA-UC materials.

\section{Experimental section}

\subsection{Materials}

Palladium(II) tetraphenylporphyrin (PdTPP) was synthesized as previously reported. ${ }^{5}$ Acrylic acid, sodium hydrate, isopropyl alcohol, potassium peroxodisulfate and toluene were purchased from Tianjin Bodi Chemical co. Tween-20, 9,10-diphenylanthracene (DPA), $\mathrm{H}_{2} \mathrm{PtCl}_{6} \cdot \mathrm{H}_{2} \mathrm{O}$ and nano powder $\mathrm{WO}_{3}$ were purchased from J\&K Chemical technology co. Methanol, coumarin and ethyl alcohol were purchased from Sinopharm Reagent Co. All reagents were analytical reagents and used as received without further purification. Deionized water was used in the experiments throughout.

\subsection{Preparation of WAR UC materials}

An amount of $36 \mathrm{~mL}$ deionized water was added into a $150 \mathrm{~mL}$ three-necked flask, and the flask was then bubbling nitrogen by a $\mathrm{N}_{2}$-vent needle for $40 \mathrm{~min}$. $10 \mathrm{~mL}$ Tween-20 was then added into the flask with syringe and dissolved in deionized water with magnetic stirring. After that, $4 \mathrm{~mL}$ mixed toluene solution contained PdTPP (0.12 mM) and DPA (36 mM) was added into the flask with syringe. The mixed solution was magnetic stirred under nitrogen atmosphere to form a homogeneous microemulsion. The microemulsion was static down to transparency and sealed for further experiment.

PAAS WAR was prepared using acrylic acid as the monomer and potassium persulfate as the initiator. $8 \mathrm{~g} \mathrm{NaOH}$ and $20 \mathrm{~mL}$ deionized water was added into a $250 \mathrm{~mL}$ three-necked flask. When the $\mathrm{NaOH}$ was dissolved with magnetic stirring and the solution cool down, $25 \mathrm{~mL}$ acrylic acid was slowly added in with magnetic stirring in cold bath. Then $20 \mathrm{~mL}$ isopropyl alcohol was added in and the solution was transferred to a water bath of $70{ }^{\circ} \mathrm{C}$. After that, $5 \mathrm{~mL}$ potassium peroxodisulfate $(40 \mathrm{mM})$ was dropwise added through dropping funnel with constant pressure. After becoming viscous, the solution was transferred into a $150 \mathrm{~mL}$ beaker and the reaction continued in a constant temperature oven of $50{ }^{\circ} \mathrm{C}$ for $2 \mathrm{~h}$. As the polymerization finished, raw PAAS was obtained as a faint yellow solid. Before applied in the next section, PAAS solid was immersed in ethyl alcohol for $2 \mathrm{~h}$ and then dried, placed in drying oven for further experiment.

$10 \mathrm{~g}$ dried PAAS was put into the prepared UC O/W microemulsion and soaked for about $48 \mathrm{~h}$. The whole process was operated in a glovebox and the quantity of water and oxygen were both below $10 \mathrm{ppm}$. After the microemulsion was well adsorbed, the surface of PAAS WAR UC materials was dried by nitrogen flow and then kept in atmospheric environment before further tests and applications.

\subsection{Upconversion property measurements}

Excitation source for UC was a solid-state continuous laser $\left(\mathrm{Nd}: \mathrm{YVO}_{4}+\mathrm{KTP}\right)$ with wavenumber of $532 \mathrm{~nm}$ and power density of $60 \mathrm{~mW} \mathrm{~cm}{ }^{-2}$. First, a mount of mixed toluene solution contains PdTPP (0.12 mM) and DPA (36 mM) was put into a quartz colorimetric ware and bubbling nitrogen by a $\mathrm{N}_{2}$-vent needle for $40 \mathrm{~min}$. Then the green laser $\left(532 \mathrm{~nm}, 60 \mathrm{~mW} \mathrm{~cm}{ }^{-2}\right)$ irradiated at the solution in the quartz colorimetric ware and the blue upconverted fluorescence appeared. ${ }^{37}$ Spectrum of the UC fluorescence was recorded by spectral scanning colorimeter (PR-655) with a $532 \mathrm{~nm}$ optical filter to get rid of the scattered laser peak.

\subsection{Photocatalytic synthesis experiment driven by WAR UC materials}

Preparation of photocatalyst $\mathrm{Pt} / \mathrm{WO}_{3}$ was as follows: $130 \mathrm{mg}$ $\mathrm{WO}_{3}$ nano powder, $30 \mathrm{~mL}$ deionized water and $10 \mathrm{~mL}$ methanol were put in a $100 \mathrm{~mL}$ beaker with ultrasonic treated for $30 \mathrm{~min}$. Then $\mathrm{H}_{2} \mathrm{PtCl}_{6} \cdot \mathrm{H}_{2} \mathrm{O}\left(0.4 \mathrm{~mL}, 5 \times 10^{-2} \mathrm{~mol} \mathrm{~L}^{-1}\right)$ was added in the beaker with stirring. After evenly stirred, the beaker was packaged by foil, irradiated under mercury lamp $(500 \mathrm{~W})$. The obtained solid was then filtered, washed with deionized water and absolute ethyl alcohol, dried and compounded as aqueous solution $\left(10 \mathrm{~g} \mathrm{~L}^{-1}\right)$ for further experiment.

Application demonstration of photocatalytic synthesis driven by WAR UC materials was as follows. $5 \mathrm{~mL}$ coumarin aqueous solution (200 $\mathrm{mg} \mathrm{L}^{-1}$ ), $0.6 \mathrm{~mL}$ prepared photocatalyst $\mathrm{Pt} / \mathrm{WO}_{3}\left(10 \mathrm{~g} \mathrm{~L}^{-1}\right)$ were stirred evenly in a $100 \mathrm{~mL}$ beaker, and then $1.5 \mathrm{~g}$ WAR UC materials was taken in the mixed solution. Green laser $\left(532 \mathrm{~nm}, 60 \mathrm{~mW} \mathrm{~cm}{ }^{-2}\right.$ ) irradiated directly at WAR UC materials and the obtained blue UC fluorescence could irradiate at coumarin aqueous solution consequently. The beaker was also packaged by foil to reduce the loss of UC fluorescence. The solution was extracted every $20 \mathrm{~min}$ to detect the fluorescence spectrum of 7-hydroxycoumarin. All the experiments were carried out in ambient environment.

\subsection{Photocatalytic degradation experiment driven by PAAS WAR UC materials}

ZnCdS powder was used as the photocatalyst candidates since its absorption edges at 528-573 $\mathrm{nm}$ and the estimated bandgaps at 2.35-2.17 eV that could effectively absorb our green-toblue upconversion (436 nm, $2.84 \mathrm{eV}$ ) to form electron/hole $\left(\mathrm{e}^{-} / \mathrm{h}^{+}\right)$pairs, ${ }^{38}$ which made it be able to degrade rhodamine $\mathrm{B}$.

Application demonstration of photocatalytic degradation driven by WAR UC materials was as follows: $5 \mathrm{~mL}$ rhodamine $\mathrm{B}$ aqueous solution $(10 \mu \mathrm{M}), 25 \mathrm{mg} \mathrm{ZnCdS}$ powder were stirred evenly in a $100 \mathrm{~mL}$ beaker, and then $1.5 \mathrm{~g}$ WAR UC materials was taken in the mixed solution. Green laser $\left(532 \mathrm{~nm}, 60 \mathrm{~mW} \mathrm{~cm}^{-2}\right)$ irradiated directly at WAR UC materials and the obtained blue 
UC fluorescence could irradiate at rhodamine B aqueous solution consequently. The beaker was also packaged by foil to reduce the loss of UC fluorescence. The solution was extracted every 20 min to detect the absorption fluorescence spectra of rhodamine B. All the experiments were carried out in ambient environment.

\section{Results and discussion}

In this paper, PdTPP was used as the sensitizer and DPA as the annihilator to constitute TTA-UC system. As metalloporphyrin complex, PdTPP showed a Soret absorption band at $415 \mathrm{~nm}$ and a Q-band at 510-550 nm (Fig. 1a), which determined the wavelength of the exciting laser $(532 \mathrm{~nm})$. There were two emission characteristic peaks as the fluorescence at $610 \mathrm{~nm}$ and phosphorescence at $710 \mathrm{~nm}$, respectively (Fig. 1a).

Absorption of DPA appeared respectively at $324 \mathrm{~nm}, 341 \mathrm{~nm}$, $359 \mathrm{~nm}$ and $378 \mathrm{~nm}$ that belonged to the vibration of anthracene group of DPA (Fig. 1b). Excited by laser of $532 \mathrm{~nm}$, emission characteristic peak showed at $400-450 \mathrm{~nm}$, which revealed DPA was an appropriate luminescent material.

The preparation process of PAAS WAR is as follows. Firstly, sodium acrylate was prepared through neutralization reaction of acrylic acid and sodium hydroxide. Secondly, sodium acrylate monomers combined with free radicals that obtained by thermal decomposition of the initiator potassium peroxodisulfate to realize chain growth. Thirdly, isopropyl alcohol was added as chain transfer agent while PAAS grew into long

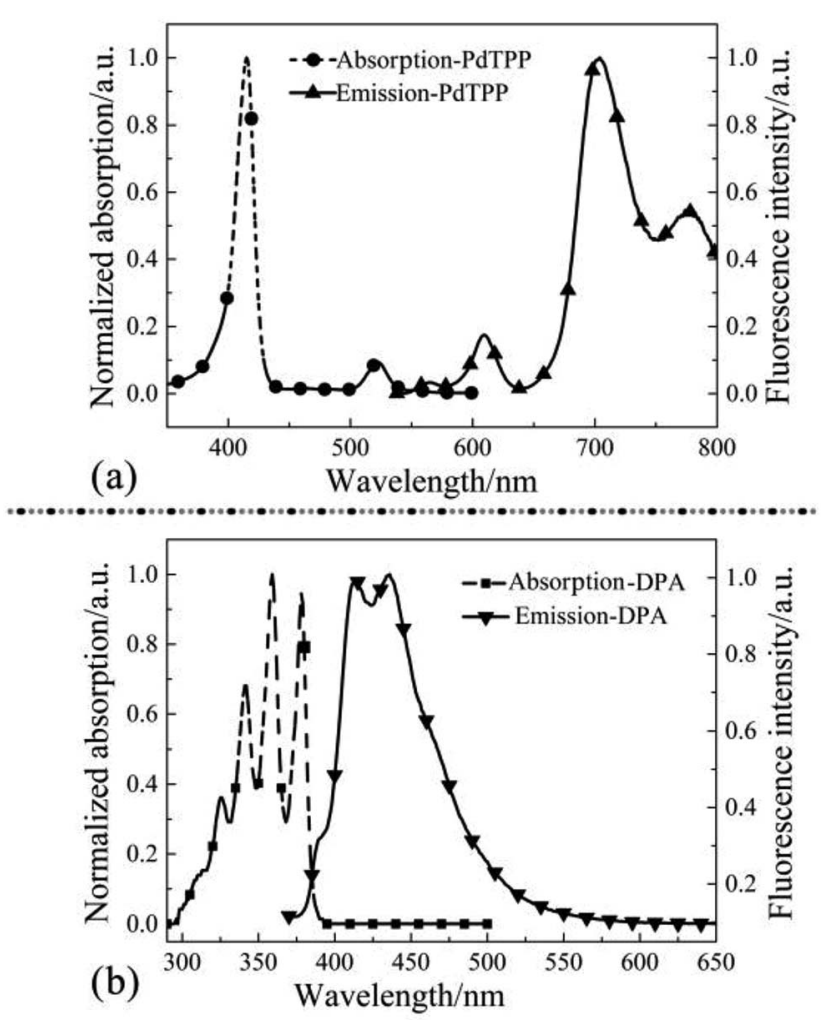

Fig. 1 Absorption and fluorescence spectra of PdTPP (a) and DPA (b) with solvent toluene at a concentration of $1 \times 10^{-6} \mathrm{~mol} \mathrm{~L}^{-1}$. chain macromolecular polymer. The prepared PAAS resin was faint yellow solid gel (Fig. 2a). As shown in SEM image (Fig. 2b), porous microstructures can be seen bestrewed within the PAAS resin, which made PAAS WAR excellent absorption performance. The water absorbency was $400 \mathrm{~mL} \mathrm{~g}^{-1}$ (eqn (S8), ESI $\dagger$ ). Therefore, the microemulsion can be absorbed into PAAS resin to form WAR UC materials.

Micro-droplets of PdTPP/DPA/toluene homogeneously dispersed in PAAS resin along with $\mathrm{O} / \mathrm{W}$ microemulsion as seen in Fig. 2d. PdTPP and DPA molecules can move unrestrictedly as in solution system, which made the energy transfer smoothly as seen in Fig. 2c. Excited by laser of $532 \mathrm{~nm}$, there revealed obvious blue light path in WAR material, which showed a high efficiency as in solution system with anaerobic treatment. All the experiments were carried out in the ambient environment, which revealed that WAR UC materials had solved the problems of oxygen-quenching and low UC efficiency in solid-state system.

Absorption and emission properties of PAAS matrix in range of 300-800 nm were shown in Fig S1a and b (ESI $\dagger$ ), and there was no absorption and emission peaks showed up. The transmittance of PAAS WAR/microemulsion system has been characterized in Fig S1c. $\uparrow$ The corrections have been applied to estimate the effect of the light loss in PAAS WAR/microemulsion system (details in ESI†). Relationship of TTA-UC intensity and excitation light power density had been explored in Fig. 3. The intensity of WAR UC materials enhanced as the increase of excitation light power density (Fig. 3a). Fig. 3b showed logarithmic plot of TTA-UC intensity versus excitation light power
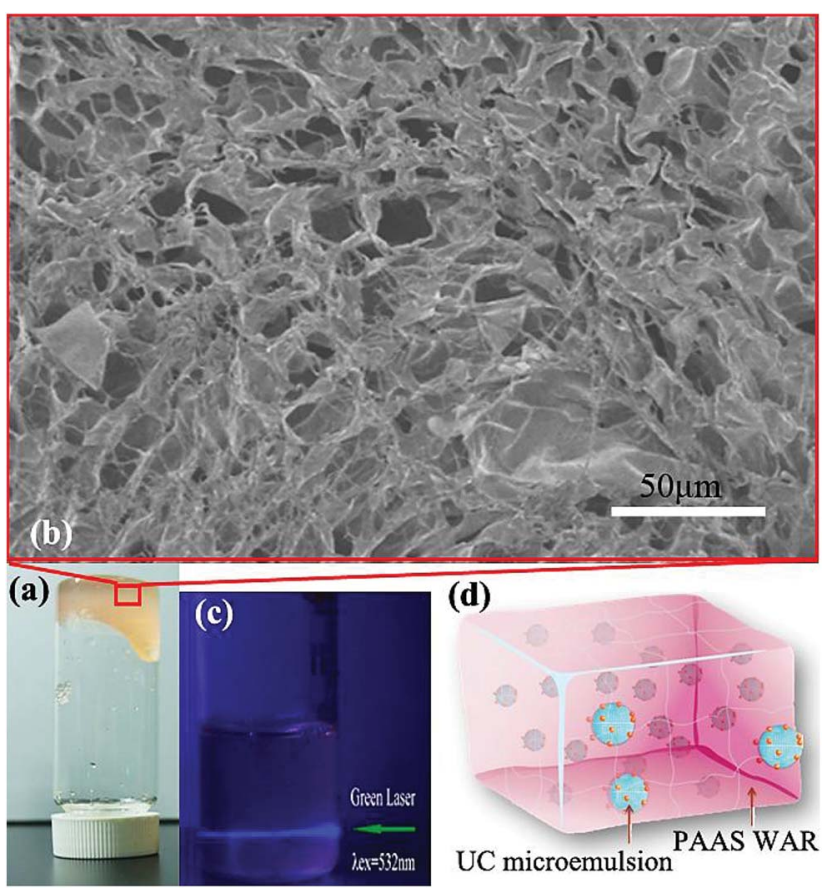

Fig. 2 (a) Photograph of WAR UC materials; (b) microstructure of WAR UC materials; (c) photograph of UC fluorescence of WAR materials; (d) schematic diagram of UC microemulsion in microcellular of WAR UC materials. 


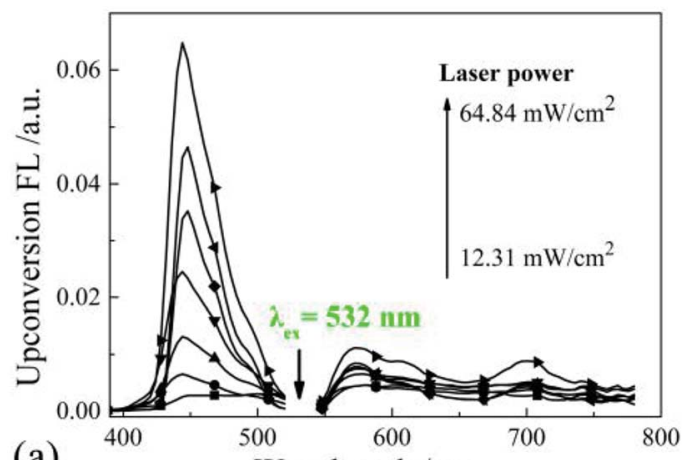

(a)

Wavelength $/ \mathrm{nm}$

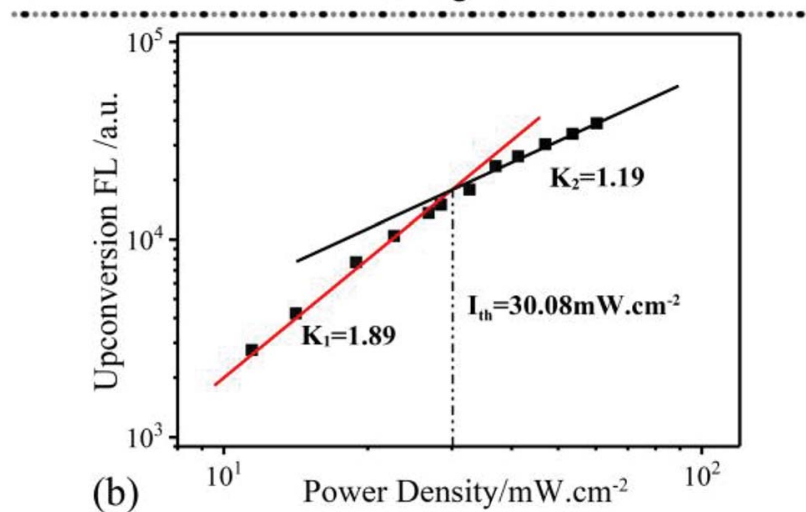

Fig. 3 (a) Spectrum of UC intensity under different excitation light power density of WAR UC materials; (b) logarithmic plots of upconversion intensity versus excitation light power density of WAR UC materials.

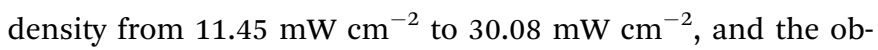
tained $K_{1}$ value is 1.89 that closed to 2 . The value represents two photons absorption of the TTA-UC process and the maximum efficiency has been reached. When the excitation light power density were increased from $30.08 \mathrm{~mW} \mathrm{~cm}^{-2}$ to $60.3 \mathrm{~mW} \mathrm{~cm}^{-2}$, the obtained $K_{2}$ value is 1.19 that closed to 1 . The value of 1.19 suggests that the two photons absorption process has achieved a saturated situation and efficiency attenuation of the TTA-UC process may occur. ${ }^{39}$ Thus, the excitation power in our upconversion measurements should be limited below $30.08 \mathrm{~mW}$ $\mathrm{cm}^{-2}$. The relationship of the WAR UC materials was the same as UC in solution system, which indicated that the PAAS resin could provide analogous condition for TTA-UC as solution system. The efficiency of WAR UC under different temperature has been studied in Fig. S2 (ESI†े).

Conventional methods for the production of 7-hydroxycoumarin may cause formation of by-products and introduces corrosion problems. For these reasons, some attempts should be taken to find alternative environmentally benign and heterogeneously catalyzed synthesis routes. ${ }^{40}$ The TTA-based upconversion can be obtained at very low excitation energy and even near-infrared light, which has a much higher penetration depth through various media, notably biological tissue. ${ }^{41}$ TTA-UC has the ability to transform the unused low energy radiation into high energy photons that beyond energy gaps of main commercial photocatalysts, and consequently trigger the chemical synthesis, which shows potential applications in photovoltaics and photocatalysis. Here, the WAR UC materials was firstly used to drive photocatalytic synthesis of organic molecules in air. Photocatalytic synthesis is a technology that using illuminant as a chemical synthesis element to substitute chemical reagent or synthesis condition, which supplied an environmentally friendly way for conventional chemical synthesis. ${ }^{42}$

In this paper, WAR UC fluorescence was used as illuminant for the photocatalytic synthesis of 7-hydroxycoumarin using coumarin as raw material. Emission characteristic peaks of coumarin and 7-hydroxycoumarin were at $375 \mathrm{~nm}$ and $460 \mathrm{~nm}$ as shown in Fig. 4a. The initial concentration of coumarin is two orders of magnitude larger than 7-hydroxycoumarin, which made the spectra intensity of coumarin is nearly constant as shown in Fig. 4a. There was no fluorescence peak at $460 \mathrm{~nm}$ at the beginning, while this fluorescence peak increased as illumination time increasing. This phenomenon demonstrated that 7-hydroxycoumarin was generated though the photocatalytic synthesis, and its concentration in solution was increasing as the illumination of WAR UC fluorescence continued. The photocatalytic synthesis of 7-hydroxycoumarin
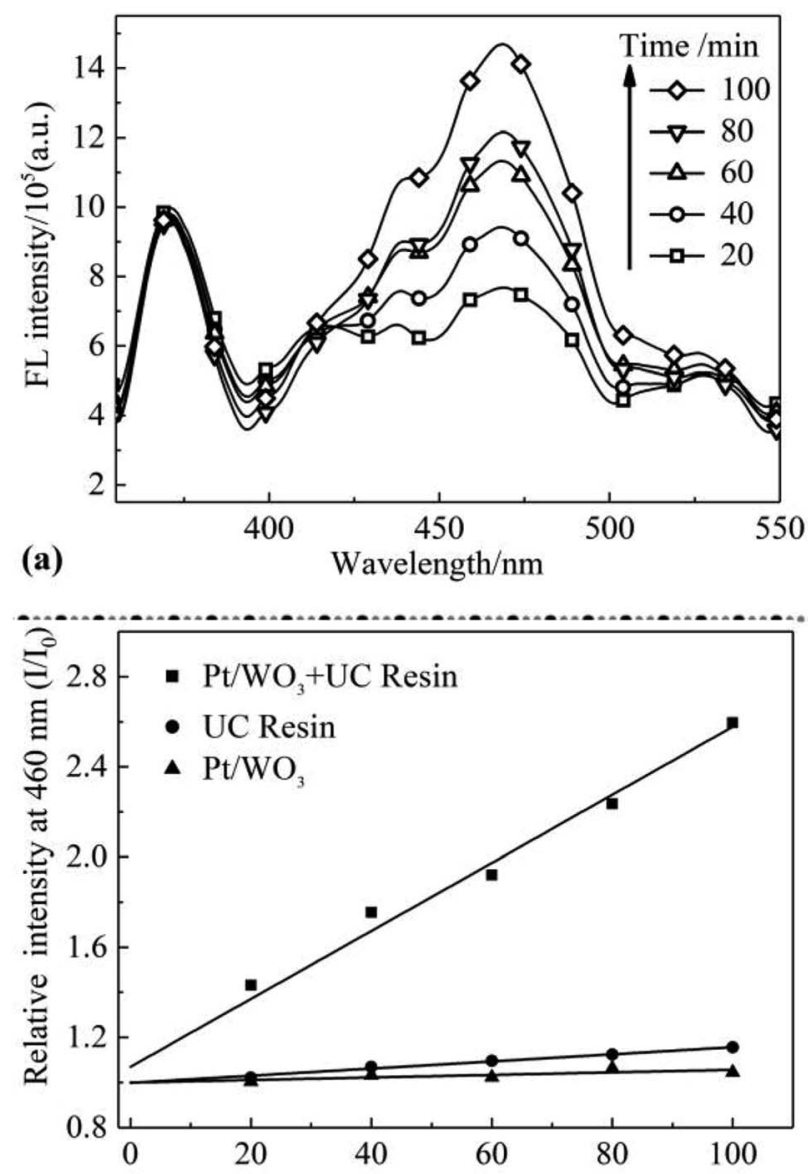

(b)

Time $/ \mathrm{min}$

Fig. 4 (a) Fluorescence spectrum of 7-hydroxycoumarin under different irradiation time; (b) conversion rate of coumarin under different conditions. 
needed a short time to be stable at the beginning. Peaks at $413 \mathrm{~nm}$ and $435 \mathrm{~nm}$ were characteristic peaks of DPA. The vast majority of the DPA was encapsulated into the PAAS resin along with microemulsion. However, there remained small amount of the DPA molecules in the outermost layer of PAAS resin, which could diffuse into coumarin solution. Emission spectra of DPA and 7-hydroxycoumarin tested using laser (365 nm) was shown in Fig. S3 (ESI $\dagger$ ). The spectra intensity of DPA at $420 \mathrm{~nm}$ came closer to the constant after $20 \mathrm{~min}$, suggesting the concentration of DPA in solution had already balanced. And the effect of DPA could be considered as the same because the spectra intensity of DPA was constant all along the photocatalytic synthesis of 7-hydroxycoumarin (Fig. 4a).

The mechanism of the photocatalytic synthesis was as follow: Firstly, low energy green light was converted into blue light of high energy that matched the band gap of $\mathrm{Pt} / \mathrm{WO}_{3}$. Then electrons of $\mathrm{Pt} / \mathrm{WO}_{3}$ was excited and transferred from valence band to conduction band. Secondly, the electrons on conduction band reacted with dissolved oxygen in solution and generated superoxide anion and hydroxyl radical. ${ }^{43}$ The hydroxyl radical on $\mathrm{Pt} / \mathrm{WO}_{3}$ is mainly generated through the reduction of $\mathrm{O}_{2}$. The charge carriers on conduction band reacted with dissolved oxygen in solution and generated $\cdot \mathrm{O}_{2}{ }^{-}$ radical. Then $\mathrm{H}_{2} \mathrm{O}_{2}$ was generated by the reaction between $\cdot \mathrm{O}_{2}{ }^{-}$ radical and $\mathrm{H}^{+}$. And $\mathrm{H}_{2} \mathrm{O}_{2}$ was reduced by electrons and splited into hydroxyl radical and $\mathrm{OH}^{-}$. The electron of $\mathrm{OH}^{-}$can be despoiled by electron hole and also transferred into hydroxyl radical. The reaction equations (eqn (S13)-(S20)) are shown in ESI. $\dagger$ Coumarin combined with the hydroxyl radical and converted into 7-hydroxycoumarin.

Single factor groups of photocatalyst and UC fluorescence were established to determine the dominant factor. Under the same condition, one group without WAR while the other without $\mathrm{Pt} / \mathrm{WO}_{3}$ (Fig. 4b). The ratio of fluorescence intensity of 7-hydroxycoumarin over time and the initial intensity $\left(I / I_{0}\right)$ was calculated as conversion rate. As seen in Fig. 4 b, the conversion rates of 7-hydroxycoumarin in the two single factor groups were nearly constant. While only the two factors of photocatalyst and UC fluorescence coexisted, the conversion rates of 7-hydroxycoumarin increased and was proportional to time. The efficiency of photocatalytic synthesis of 7-hydroxycoumarin was also obtained (eqn (S12), ESI $\dagger$ ).

Another demo application of WAR UC materials in the photocatalytic degradation were demonstrated. Rhodamine $\mathrm{B}$ is a mature industrial dye that has been widely applied in cosmetics and colored glass field. However, rhodamine B is carcinogenic and its industrial or domestic waste may be threats for human health. Using the UC fluorescence as illuminant, we tried to study the photocatalyst degradation behavior of rhodamine B by WAR UC materials. Schematic diagram of the photocatalytic degradation of rhodamine B was shown in Fig. 5a. Semiconductor materials ZnCdS was used as photocatalyst. Under the irradiation of $532 \mathrm{~nm}$ laser, PdTPP and DPA emitted high-energy UC fluorescence that exciting ZnCdS to generate electric charge effect. Through the electric charge effect, rhodamine B was gradually degraded.
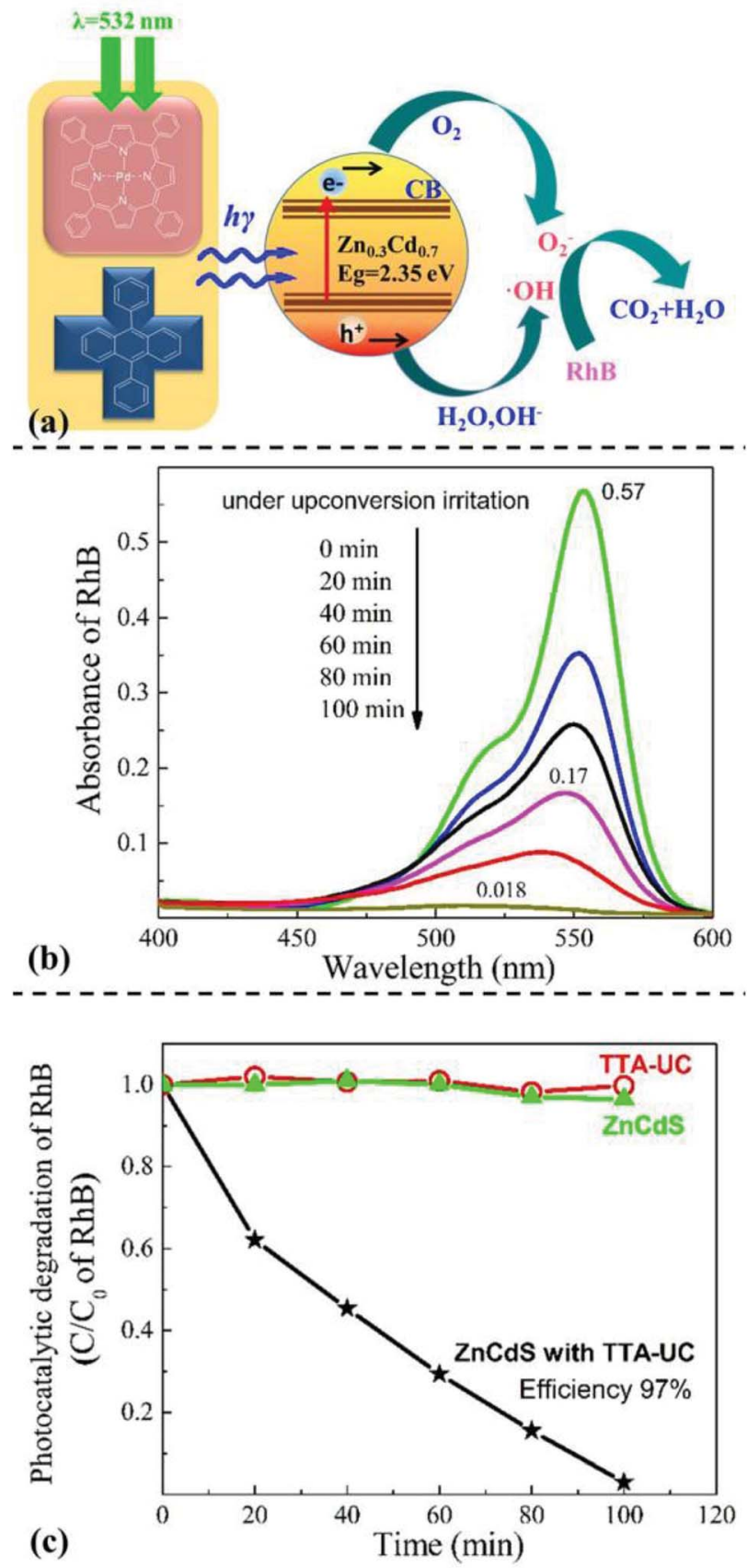

Fig. 5 (a) Schematic diagram of photo-degradation test of WAR UC materials/ZnCdS/rhodamine B; (b) absorption fluorescence spectra of rhodamine $B$ under different irradiation time; (c) photo-degradation curves of rhodamine $B$ under different conditions.

Absorption spectrum of rhodamine B solution varied over time under the irradiation of WAR UC fluorescence as shown in Fig. 5b. Absorbance intensity of rhodamine B gradually declined along with the irradiation of UC fluorescence, which means the concentration of rhodamine B declined gradually. The initial absorbance of rhodamine $B$ was 0.57 , while the value turned to 0.018 after illumination for $10 \mathrm{~min}$. The concentration of rhodamine B had decreased for almost 97\%, which indicated 
a remarkable photocatalytic degradation of rhodamine $\mathrm{B}$ through WAR UC materials.

Single factor groups of photocatalyst and UC fluorescence were also established to determine the dominant factor. Under the same condition, one group without WAR while the other without ZnCdS, as shown in Fig. 5c. The relative concentration of rhodamine B in the two single factor groups were nearly constant. While only the two factors of photo catalyst and UC fluorescence coexisted, the relative concentration of rhodamine B decreased distinctly over time. The degradation efficiency was up to $97 \%$ that corresponded to the result of ultraviolet absorption test. The mechanism of the degradation of rhodamine B was as follows. Firstly, low energy exciting light was converted into blue UC fluorescence of high energy through WAR. Secondly, the energy of blue UC fluorescence beyond the energy difference of valence band and conduction band of ZnCdS, which generated electron holes and electrons. And these charge carriers on conduction band reacted with dissolved oxygen in solution and generated hydroxyl radical in the same way of $\mathrm{Pt} / \mathrm{WO}_{3}$. The reaction equations are shown in ESI. $\dagger$ Thirdly, rhodamine B was gradually degraded into $\mathrm{CO}_{2}$ and $\mathrm{H}_{2} \mathrm{O}$ by hydroxyl radical following a series of redox processes. The remarkable photocatalytic degradation of rhodamine $B$ through WAR material demonstrated the potential in industrial applications of photocatalytic degradation field.

\section{Conclusions}

In this paper, water-absorbent resin (WAR) solid-state upconversion (UC) material based on triplet-triplet annihilation mechanism was prepared by loading PdTPP/DPA as sensitizers and annihilators, using polyporous sodium polyacrylate as matrix. PdTPP/DPA was dissolved in toluene and prepared into $\mathrm{O} / \mathrm{W}$ microemulsion. Characterization of micro-morphology revealed that porous microstructures of the PAAS resin absorbed the microemulsion to form WAR UC materials. UC property showed that PAAS WAR UC materials revealed strong blue emission under the simulation of a semiconductor laser (532 $\mathrm{nm}, 60 \mathrm{~mW} \mathrm{~cm}^{-2}$ ), realizing highly efficient solid-state TTA-UC in air. In the further application measurement, photocatalyst $\mathrm{Pt} / \mathrm{WO}_{3}$ was excited by UC emission from WAR UC materials to produce hydroxyl radicals, yielding 7-hydroxycoumarin by reacted with coumarin. Similarly, the band gap of ZnCdS matched the energy of UC emission, hole-electron pairs were obtained under the UC irradiation and captured electrons from rhodamine $\mathrm{B}$, leading to the degradation of rhodamine $\mathrm{B}$. The maximum photocatalysis efficiency was up to $97 \%$.

This work solves the oxygen quenching problem and opens a new avenue to solid-state devices for TTA-UC by the prepared WAR UC materials. Furthermore, the applications of photocatalytic synthesis and photocatalytic degradation lay a foundation for future practical applications for TTA-UC materials.

\section{Conflicts of interest}

There are no conflicts to declare.

\section{Acknowledgements}

The authors are grateful to National Natural Science Foundation of China (Grant No. 51873145, 51603141), Natural Science Foundation of Jiangsu Province-Excellent Youth Foundation (BK20170065), Natural Science Foundation of Jiangsu Province (BK20160358), Natural Science Foundation of the Higher Education Institutions of Jiangsu Province (17KJA430016), Qing Lan Project, 5th 333 High-level Talents Training Project of Jiangsu Province (No. BRA2018340), Six Talent Summits Project of Jiangsu Province (No. XCL-79) for the financial supports.

\section{References}

1 S. L. Chen, J. Stehr, N. K. Reddy, C. W. Tu, W. M. Chen and I. A. Buyanova, Appl. Phys. B: Lasers Opt., 2012, 108, 919.

2 W. Dong, G. Zhou, X. Xu, X. Wang, Z. Liu, R. Yan, Z. Shao and M. Jiang, Opt. Laser Technol., 2002, 34, 55.

3 Z. Hasan, L. Biyikli, M. J. Sellars, G. A. Khodaparast, F. S. Richardson and J. R. Quagliano, Phys. Rev. B: Condens. Matter Mater. Phys., 1997, 56, 4518.

4 C. Ye, L. Zhou, X. Wang and Z. Liang, Phys. Chem. Chem. Phys., 2016, 18, 10818.

5 W. Bao, B. Sun, X. Wang, C. Ye, D. Ping, Z. Liang, Z. Chen, X. Tao and L. Wu, J. Phys. Chem. C, 2014, 118, 1417.

6 X. Ming, X. Zou, Q. Su, Y. Wei, C. Cong, Q. Wang, X. Zhu, F. Wei and F. Li, Nat. Commun., 2018, 9, 2698.

7 Q. Dou, L. Jiang, D. Kai, C. Owh and J. L. Xian, Drug Discovery Today, 2017, 22, 1400.

8 Y. T. Liang, B. K. Vijayan, K. A. Gray and M. C. Hersam, Nano Lett., 2011, 11, 2865.

9 W. Fan, H. Bai and W. Shi, CrystEngComm, 2014, 16, 3059.

10 Y. Yi, L. Cheng, M. Ping and L. J. Wang, J. Nanomater., 2013, 2013, 13.

11 Y. Chen, J. Zhao, L. Xie, H. Guo and Q. Li, RSC Adv., 2012, 2, 3942.

12 T. Jing, Y. Ni, C. Lu, C. Jie, Y. Yuan, J. Chen and Z. Xu, RSC Adv., 2014, 4, 39316.

13 N. M. Idris, M. K. Gnanasammandhan, J. Zhang, P. C. Ho, R. Mahendran and Y. Zhang, Nat. Med., 2012, 18, 1580.

14 C. Wang, L. Cheng, Y. Liu, X. Wang, X. Ma, Z. Deng, Y. Li and Z. Liu, Adv. Funct. Mater., 2013, 23, 3077.

15 H. Ling, Y. Zhao, H. Zhang, K. Huang, J. Yang and G. Han, Angew. Chem., Int. Ed. Engl., 2017, 56, 14400.

16 T. Miteva, V. Yakutkin, G. Nelles and S. Baluschev, New J. Phys., 2008, 10, 103002.

17 C. Ye, J. Wang, X. Wang, P. Ding, Z. Liang and X. Tao, Phys. Chem. Chem. Phys., 2016, 18, 3430.

18 K. Xu, J. Zhao and E. G. Moore, J. Phys. Chem. C, 2015, 121, 22665.

19 D. Liu, Y. Zhao, Z. Wang, K. Xu and J. Zhao, Dalton Trans., 2018, 47, 8619.

20 Q. Li, C. Zhang, J. Y. Zheng, Y. S. Zhao and J. Yao, Chem. Commun., 2011, 48, 85.

21 K. Xu, Z. Mahmood, D. Escudero, J. Zhao and D. Jacquemin, J. Phys. Chem. C, 2015, 119, 23801. 
22 T. Yang, Y. Sun, Q. Liu, W. Feng, P. Yang and F. Li, Biomaterials, 2012, 33, 3733.

23 Z. Mahmood, A. Toffoletti, J. Zhao and A. Barbon, J. Lumin., 2017, 183, 507.

24 H. Sternlicht, G. C. Nieman and G. W. Robinson, J. Chem. Phys., 1963, 38, 1326.

25 D. Dzebo, K. Mothpoulsen and B. Albinsson, Photochem. Photobiol. Sci., 2017, 16, 1327.

26 Z. Xiang, S. Shikha and Z. Yong, Nanoscale, 2018, 10, 16447.

27 S. H. C. Askes, V. C. Leeuwenburgh, W. Pomp, H. Arjmanditash, S. Tanase, T. Schmidt and S. Bonnet, ACS Biomater. Sci. Eng., 2017, 3, 322.

28 C. Ye, B. Wang, X. Wang, P. Ding, X. Tao, Z. Chen, Z. Liang and Y. Zhou, J. Mater. Chem. C, 2014, 2, 8507.

29 X. Jiang, X. Guo, J. Peng, D. Zhao and Y. Ma, ACS Appl. Mater. Interfaces, 2016, 8, 11441.

30 Y. Y. Cheng, A. Nattestad, T. F. Schulze, R. W. Macqueen, B. Fückel, K. Lips, G. G. Wallace, T. Khoury, M. J. Crossley and T. W. Schmidt, Chem. Sci., 2015, 7, 559.

31 H. Yonemura, Y. Naka, M. Nishino, H. Sakaguchi and S. Yamada, Mol. Cryst. Liq. Cryst., 2017, 654, 196.

32 S. Baluschev, P. E. Keivanidis, G. Wegner, J. Jacob, A. C. Grimsdale, K. Mullen, T. Miteva, A. Yasuda and G. Nelles, Appl. Phys. Lett., 2005, 86, 3065.
33 P. B. Merkel and J. P. Dinnocenzo, J. Lumin., 2009, 129, 303. 34 L. Li, Y. Zeng, T. Yu, J. Chen, G. Yang and Y. Li, Chemsuschem, 2017, 10, 4610.

35 R. Vadrucci, A. Monguzzi, F. Saenz, B. D. Wilts, Y. C. Simon and C. Weder, Adv. Mater., 2017, 29, 1702992.

36 L. Nienhaus, M. Wu, V. Bulovia, M. A. Baldo and M. G. Bawendi, Dalton Trans., 2018, 47, 8509.

37 R. Salhi and J. L. Deschanvres, J. Lumin., 2016, 176, 250.

38 K. S. Kulkarin, D. Winkler, H. P. Borse and R. Fink, Appl. Surf. Sci., 2001, 169, 438.

39 A. Haefele, J. Blumhoff, R. S. Khnayzer, et al., J. Phys. Chem. Lett. , 2012, 3, 299.

40 M. C. Laufer, H. Hausmann and W. F. Hölderich, J. Catal., 2003, 218, 315.

41 D. Benjamin Ravetz, B. P. Andrew, M. C. Emily, et al., Nature, $2019,343$.

42 H. Kim, S. Weon, H. Kang, A. L. Hagstrom, O. S. Kwon, Y. S. Lee, W. Choi and J. H. Kim, Environ. Sci. Technol., 2016, 50, 11184.

43 J. Kim, C. W. Lee and W. Choi, Environ. Sci. Technol., 2010, 44, 6849. 